\title{
The implication of ocular manifestation of COVID-19 for medical staff and patients - systematic review
}

The Editors of the journal Annals of Agricultural and Environmental Medicine inform that in the article The implication of ocular manifestation of COVID-19 for medical staff and patients - systematic review by Latalska Małgorzata, Mackiewicz Jerzy (Ann Agric Environ Med. 2020; 27(2): 165-170. doi: 10.26444/aaem/122790), in Table 2, the article by Chen L, Deng $\mathrm{C}$, Chen X et al. Ocular manifestations and clinical characteristics of 534 cases of COVID19 in China: A cross-sectional study. medRxiv 2020 (doi.org/10.1101/2020.03.12.20034678) was incorrectly quoted.

The printed version is

Chen et al, 2020 [16].

This is incorrect and should be Chen et al, 2020 [29]. 